\title{
Wendroff type integro-sum inequalities and applications
}

\author{
Anatoly Samoilenko, Sergiy Borysenko, Ettore \\ Laserra, and Giovanni Matarazzo
}




\title{
WENDROFF TYPE INTEGRO-SUM INEQUALITIES AND APPLICATIONS
}

\author{
Anatolij Samoilenko \\ Institute of Mathematics, Ukrainian National Academy of Sciences \\ 3 Tereshenkovskaya Str., 01601 KIEV, Ukraine \\ sam@imath.kiev.ua \\ Sergiy Borysenko \\ Department of Mathematics, National Technical University of Ukraine \\ 37 Peremohy Str., 03056 KIEV, Ukraine \\ borys@mbox.com.ua \\ ETTORE LASERRA \\ Department of Mathematics and Informatics, University of Salerno, Italy \\ S. Allende Str., 84081 BARONISSI, Italy \\ laserra@unisa.it \\ Giovanni Matarazzo \\ Department of Engineering and Applied Mathematics, University of Salerno, Italy \\ S.Allende Str., 84081 BARONISSI, Italy \\ matarazzo@bridge.diima.unisa.it
}

[Received: June 20, 2002]

\begin{abstract}
We present some new nonlinear Wendroff type integral inequalities with respect to two independent variables for discontinuous functions. The results obtained are applied to investigate properties of solutions of a certain class of hyperbolic equations with impulse influence on certain surfaces.
\end{abstract}

Mathematical Subject Classification: 34B15

Keywords: differential equations with impulse influence, integro-sum inequalities

\section{Introduction}

Differential models of various real processes are described by systems of ordinary differential equations with impulse influence [1-3]. Among the methods of investigating the properties of these systems (such as existence, uniqueness, boundedness, and stability of solutions), the method of integro-sum inequalities [4-15] plays an important role. Note that applications and generalizations of this method to estimate solutions of systems of partial differential equations with impulse perturbations were not considered. 
The study of this kind of problems is motivated by models of real processes in the dynamics of hydromechanical systems which are described by certain classes of hyperbolic partial differential equations with impulse perturbations, where the impulse effect is concentrated on surfaces transversal to characteristics.

Integro-sum representations of the solutions of such systems contain LebesgueStiltjes measure concentrated on the curves of jumps of the solutions.

\section{Main results}

Let us assume that:

(a) $D^{*}$ is an open set in $R^{2}\left(D^{*} \subset R^{2}\right)$;

(b) $D^{*}=D \backslash \Gamma$, where $D=\bigcup_{j} D_{j}, j=1,2, \ldots$;

(c) $\Gamma=\bigcup_{j} \Gamma_{j}, \Gamma_{j}=\left\{(x, y): \varphi_{j}(x, y)=0, j=1,2, \ldots\right\}$;

(d) $\varphi_{j}(x, y)$ are real-valued continuously differentiable functions such that $\operatorname{grad} \varphi_{j}(x, y)>$ 0 for all $j=1,2, \ldots$;

(e) $D_{1} \stackrel{\text { def }}{=}\left\{(x, y): x \geq 0, y \geq 0, \varphi_{1}(x, y)<0\right\}$; $D_{k} \stackrel{\text { def }}{=}\left\{(x, y): x \geq 0, y \geq 0, \varphi_{k-1}(x, y) \geq 0, \varphi_{k}(x, y)<0, \quad \forall k>2, k \in N\right\} ;$

(f) $G_{j}=\left\{(u, v):(x, y) \in D_{j}, 0 \leq u \leq x, 0 \leq v \leq y, j \in N\right\}$;

(g) $\mu_{\varphi_{k}}$ is the Lebesgue-Stiltjes measure concentrated on the curves $\Gamma_{k}$.

Let us consider a real-valued nonnegative continuous function $u(x, y)$ on $D^{*}$, which has finite jumps on the curves $\left\{\Gamma_{j}\right\}$.

Denote by $\Phi(x, y, u)$ a nonnegative, continuous, and nondecreasing function of $u$, with $x, y \in D^{*}$ fixed. We shall consider the functions $\Phi$ of one of the following types $\Phi=\Phi_{j}(j=1,4)$ :

(i) $\Phi_{1}(x, y, u)=f_{1}(x, y) u(x, y)$,

(ii) $\Phi_{2}(x, y, u)=f_{2}(x, y) u(x, y)+f_{3}(x, y)$,

(iii) $\Phi_{3}(x, y, u)=f_{4}(x, y) u^{\alpha}(x, y), \alpha=$ const $>0, \alpha \neq 1$,

(iv) $\Phi_{4}(x, y, u)=f_{5}(x, y) u(x, y)+f_{6}(x, y) u^{\alpha}(x, y)$, where $f_{j}(x, y)(j=\overline{1,6})$ are continuous nonnegative functions in $R_{+}^{2}=\{(x, y): x \geq 0, y \geq 0\}$.

Let $W(x, y, u(x, y))$ denote a function of one of the following two types $W_{1}$ and $W_{2}$ :

$$
W_{1}=\beta_{j} u(x, y), \quad j \in N
$$

where $\beta_{j} \geq 0$ are constants, and

$$
W_{2}=\beta_{j}(x, y) u(x, y), \quad j \in N,
$$

where $\beta_{j}(x, y)$ are continuous functions nonnegative for all $(x, y) \in R_{+}^{2}$.

Let $g(x, y)$ be a positive, nondecreasing continuous function in $R_{+}^{2}$. Assume that $u(x, y)$ satisfies the following integro-sum inequality in $D^{*}$ :

$$
u(x, y) \leq g(x, y)+\iint_{G_{n}} \Phi(\tau, s, u(\tau, s)) d \tau d s+\sum_{j=1}^{n-1} \int_{\Gamma_{j} \cap G_{n}} W(x, y, u(x, y)) d \mu_{\varphi_{j}},
$$


which is satisfied for all $(x, y) \in D^{*}$. Then the following results are true.

Proposition 1. The estimate

$$
\begin{gathered}
u(x, y) \leq g(x, y) \exp \left[F_{1}(x, y)\right] \prod\left(\beta_{j}(x, y)\right), \text { if } \Phi=\Phi_{1}, W=W_{2} \\
u(x, y) \leq g(x, y) \exp \left[F_{1}(x, y)\right] \prod\left(\beta_{j}\right), \text { if } \Phi=\Phi_{1}, W=W_{1}
\end{gathered}
$$

holds for all $(x, y) \in D^{*}$. Here,

$$
\begin{gathered}
\int_{0}^{x} \int_{0}^{y} f_{i}(\tau, s) d \tau d s \stackrel{\text { def }}{=} F_{i}(x, y), i=1,3,5 \\
\prod_{j=1}^{n-1} \int_{\Gamma_{j} \cap G_{n}}\left(1+\beta_{j}(x, y)\right) d \mu_{\varphi_{j}} \stackrel{\text { def }}{=} \prod\left(\beta_{j}(x, y)\right), \quad \prod_{j=1}^{n-1}\left(1+\beta_{j}\right) \bigvee_{\Gamma_{j} \cap G_{n}}\left(\mu_{\varphi_{j}}\right) \stackrel{\text { def }}{=} \prod\left(\beta_{j}\right),
\end{gathered}
$$

where $\bigvee_{\Gamma_{j} \cap G_{n}}\left(\mu_{\varphi_{j}}\right)$ is the complete variation of $\mu_{\varphi_{j}}$.

Proposition 2. The estimate

$$
\begin{aligned}
u(x, y) \leq g(x, y) \exp \left[F_{2}(x, y)\right] & \prod\left(\beta_{j}(x, y)\right) \times \\
& \times\left(1+\int_{0}^{x} \int_{0}^{y} \frac{f_{3}(\tau, s)}{g(\tau, s)} \exp \left[-F_{2}(\tau, s)\right] d \tau d s\right)
\end{aligned}
$$

holds if $\Phi=\Phi_{2}, W=W_{2}$, and

$$
u(x, y) \leq g(x, y) \exp \left[F_{2}(x, y)\right] \prod\left(\beta_{j}\right)\left(1+\int_{0}^{x} \int_{0}^{y} \frac{f_{3}(\tau, s)}{g(\tau, s)} \exp \left[-F_{2}(\tau, s)\right] d \tau d s\right),
$$

if $\Phi=\Phi_{2}, W=W_{1}$.

Proposition 3. The following assertions hold.

(A) The estimate

$$
u(x, y) \leq g(x, y) \prod\left(\beta_{j}(x, y)\left[1+(1-\alpha) \int_{0}^{x} \int_{0}^{y} f_{4}(\tau, s) g^{\alpha-1}(\tau, s) d \tau d s\right]^{\frac{1}{1-\alpha}}\right.
$$

is true if $0<\alpha<1, \Phi=\Phi_{3}$, and $W=W_{2}$. When $\beta_{j}(x, y)=\beta_{j}=$ const $>0$, the expression $\prod\left(\beta_{j}(x, y)\right)$ in estimate $(A)$ is replaced by $\prod\left(\beta_{j}\right)$.

(B) The estimate

$$
\begin{aligned}
u(x, y) & \leq g(x, y) \prod\left(\beta_{j}(x, y)\right)[1-(\alpha-1) \times \\
& \left.\times \prod^{\alpha-1} \beta_{j}(x, y) \int_{0}^{x} \int_{0}^{y} f_{y}(\tau, s) g^{\alpha-1}(\tau, s) d \tau, d s\right]^{-\frac{1}{\alpha-1}}
\end{aligned}
$$

holds for $\alpha>1$ and for arbitrary $(x, y) \in D^{*}$ such that

$$
\int_{0}^{x} \int_{0}^{y} f_{4}(\tau, s) g^{\alpha-1}(\tau, s) d \tau d s<\left[(\alpha-1) \prod^{\alpha-1}\left(\beta_{j}(x, y)\right)\right]^{-1} .
$$

If $\beta_{j}(x, y)=\beta_{j}=\mathrm{const}>0$, then $\prod\left(\beta_{j}(x, y)\right)$ in estimate $(B)$ is replaced by $\prod\left(\beta_{j}\right)$.

Proposition 4. The following assertions hold. 
(C) The estimate

$$
\begin{aligned}
u(x, y) & \leq g(x, y) \prod\left(\beta_{j}(x, y)\right) \exp \left[F_{5}(x, y)\right] \times\left[1+(1-\alpha) \int_{0}^{x} \int_{0}^{y} f_{6}(\tau, s) g^{\alpha-1}(\tau, s) \times\right. \\
& \left.\times \exp \left[(\alpha-1) F_{5}(\tau, s)\right] d \tau d s\right]^{\frac{1}{1-\alpha}}
\end{aligned}
$$

holds for $0<\alpha<1, \Phi=\Phi_{4}, W=W_{2}$. If $\beta_{j}(x, y)=\beta_{j}$, the expression $\prod\left(\beta_{j}(x, y)\right)$ in $(C)$ changes to $\prod\left(\beta_{j}\right)$.

(D) The estimate

$$
\begin{aligned}
u(x, y) \leq g(x, y) \prod & \left(\beta_{j}(x, y)\right) \exp \left[F_{5}(x, y)\right]\left[1-(\alpha-1) \prod^{\alpha-1}\left(\beta_{j}(x, y)\right) \times\right. \\
& \left.\times \int_{0}^{x} \int_{0}^{y} f_{6}(\tau, s) \times g^{\alpha-1}(\tau, s) \exp \left[(\alpha-1) F_{5}(\tau, s)\right] d \tau d s\right]^{-\frac{1}{\alpha-1}}
\end{aligned}
$$

is true for $\alpha>1$ and arbitrary $(x, y) \in D^{*}$ such that

$$
\begin{aligned}
& \int_{0}^{x} \int_{0}^{y} f_{6} g^{\alpha-1} \exp \left[(\alpha-1) F_{5}\right] d \tau d s<\left[(\alpha-1) \prod^{\alpha-1}\left(\beta_{j}(x, y)\right)\right]^{-1} . \\
& \text { If } \beta_{j}(x, y)=\beta_{j} \text {, the expression } \prod\left(\beta_{j}(x, y)\right) \text { in }(D) \text { changes to } \prod\left(\beta_{j}\right) \text {. }
\end{aligned}
$$

The proofs of Propositions 1-4 are obtained by using the induction method.

Proof. Let us establish Proposition 1 (Propositions 2-4 are proved analogously).

Let $(x, y) \in D_{1}$. Inequality $(2.1)$ reduces to the form

$$
u(x, y) \leq g(x, y)+\iint_{G_{1}} \Phi_{1}(\tau, s, u(\tau, s)) d \tau d s .
$$

We can suppose that $g(x, y)=C>0$, a constant function, because otherwise, if $g(x, y) \neq C$ then, by using the fact that $g$ is positive and nondecreasing, it is possible to obtain the comparison inequality

$$
\mu(x, y) \leq 1+\iint_{G_{1}} \Phi_{1}(\tau, s, \mu(\tau, s)) d \tau d s,
$$

where $\mu=\frac{u}{g}$.

Obviously, the following estimate holds in $D_{1}$ :

$$
u(x, y) \leq C \exp \left[F_{1}(x, y)\right] .
$$

Consider the domain $D_{2}$. Let $G_{2}=G_{2}^{1} \cup G_{2}^{2}$, where

$$
G_{2}^{2}=\left\{(x, y):(x, y) \in D_{2} \cap G_{2}\right\} .
$$

Then, for all $(x, y) \in D_{2}$, the inequality 


$$
\begin{aligned}
u(x, y) \leq C+\iint_{G_{2}^{1}} \Phi_{1}(\tau, s, u(\tau, s)) d \tau d s+\int_{\Gamma_{1} \cap G_{2}} \beta_{1}(x, y) u(x, y) d \mu_{\varphi_{1}} \\
\quad+\iint_{G_{2}^{2}} \Phi_{1}(\tau, s, u(\tau, s)) d \tau d s
\end{aligned}
$$

holds.

On the curves $\Gamma_{1} \cap G_{2}$, we select some points $A_{i}\left(x_{i}^{1}, y_{i}^{1}\right), i=\overline{0, n-1}$, and consider the inequality

$$
\begin{aligned}
u(x, y) \leq \sum_{i=0}^{n-1}\left(C+\int_{0}^{x_{1}^{1}} \int_{0}^{y_{i}^{1}} \Phi_{1}(\tau, s, u(\tau, s)) d \tau d s\right. & +u\left(x_{i}^{1}, y_{i}^{1}\right) \beta_{1}\left(x_{i}^{1}, y_{i}^{1}\right) \Delta \mu_{\varphi_{i}^{i}} \\
& \left.+\int_{x_{i}^{1}}^{x} \int_{y_{i}^{1}}^{y} \Phi_{1}(\tau, s, u(\tau, s)) d \tau d s\right),
\end{aligned}
$$

where $\Delta \mu_{\varphi_{1}^{i}}$ is the variation of the measure function $\varphi_{1}$ on the segment $A_{i} A_{i+1}$. Using (2.2), we obtain

$$
\begin{gathered}
u(x, y) \leq \sum_{i=0}^{n-1}\left\{C \exp \left[F_{1}\left(x_{i}^{1}, y_{i}^{1}\right)\right]+C \beta_{1}\left(x_{i}^{1}, y_{i}^{1}\right) \Delta \mu_{\varphi_{1}}^{i} \exp \left[F_{1}\left(x_{i}^{1}, y_{i}^{1}\right)\right]+\right. \\
\left.+\int_{x_{i}^{1}}^{x} \int_{y_{i}^{1}}^{y} \Phi_{1} d \tau d s\right\} \leq \sum_{i=0}^{n-1}\left\{C \left(1+\beta_{1}\left(x_{i}^{1}, y_{i}^{1}\right) \Delta \mu_{\varphi_{1}}^{i} \exp \left[F_{1}\left(x_{i}^{1}, y_{i}^{1}\right)\right]+\right.\right. \\
\left.\left.+\int_{x_{i}^{1}}^{x} \int_{y_{i}^{1}}^{y} \Phi_{1} d \tau d s\right)\right\} \leq \sum_{i=0}^{n-1}\left[C\left(1+\beta_{1}\left(x_{i}^{1}, y_{i}^{1}\right)\right) \Delta_{\varphi_{1}}^{i} \exp \left[F_{1}(x, y)\right]\right] .
\end{gathered}
$$

When $\max _{0 \leq i \leq n-1} \Delta \mu_{\varphi_{1}}^{i} \rightarrow 0$, we obtain

$$
u(x, y) \leq C \exp \left[F_{1}(x, y)\right] \int_{\Gamma_{1} \cap G}\left(1+\beta_{1}(x, y)\right) d \mu_{\varphi_{1}} .
$$

Let $(2.2)$ be satisfied for all $(x, y) \in D_{k}$. Let us consider $(x, y) \in D_{k+1}$ and put $G=G_{k+1}^{1} \cup G_{k+1}^{2}$,

$$
G_{k+1}^{1}=\left\{(x, y):(x, y) \in D_{k+1} \cap G\right\}, G_{k+1}^{2}=G \backslash G_{k+1}^{1} .
$$

Then

$$
u(x, y) \leq C+\iint_{G_{k+1}^{1}} \Phi_{1} d \tau d s+\int_{\Gamma_{k} \cap G} \beta_{k}(x, y) u(x, y) d \mu_{\varphi_{k}}+\iint_{G_{k+1}^{2}} \Phi_{1} d \tau d s .
$$

On the curve $\Gamma_{k} \cap G$, we select some points $B_{i}\left(x_{i}^{k}, y_{i}^{k}\right), i=\overline{0, n-1}$. Denote by $\Delta \mu_{\varphi_{k}}^{i}$ the variation of the measure on the segment $B_{i} B_{i+1}$. Similarly to the consideration above, we obtain

$$
u(x, y) \leq \sum_{i=0}^{n-1}\left[C+\int_{0}^{x_{i}^{k}} \int_{0}^{y_{i}^{k}} \Phi_{1} d \tau d s+u\left(x_{i}^{k}, y_{i}^{k}\right) \beta_{k}\left(x_{i}^{k}, y_{i}^{k}\right) \Delta_{\varphi_{k}}^{i}+\right.
$$




$$
\left.+u\left(x_{i}^{k}, y_{i}^{k}\right) \beta_{k}\left(x_{i}^{k}, y_{i}^{k}\right) \Delta_{\varphi_{k}}^{i}+\int_{x_{i}^{k}}^{x} \int_{y_{i}^{k}}^{y} \Phi_{1} d \tau d s\right] .
$$

Then

$$
u(x, y) \leq \sum_{i=0}^{n-1}\left[C\left(1+\beta_{k}\left(x_{i}^{k}, y_{i}^{k}\right)\right) \times \Delta \mu_{\varphi_{k}}^{i} \exp \left[F_{1}(x, y)\right] \prod_{j=1}^{n-1} \int_{\Gamma_{j} \cap G}\left(1+\beta_{j}(x, y)\right) d \mu_{\varphi_{j}}\right] .
$$

When $\max _{0 \leq i \leq n-1} \Delta \mu_{\varphi_{K}}^{i} \rightarrow 0$, we get

$$
u(x, y) \leq C \exp \left[F_{1}(x, y)\right] \prod_{j=1}^{k} \int_{\Gamma_{j} \cap G}\left(1+\beta_{j}(x, y)\right) d \mu_{\varphi_{j}}
$$

for all $(x, y) \in D_{k+1}$. This completes the proof of Proposition 1 .

\section{Applications}

Consider the model of a hydraulic system consisting of a tank, a delivery pipeline, an auger centrifugal pump, and a pressure head pipeline giving a liquid in gasogenerator and the chamber output pressure. Such systems were considered in $[16,17,20]$. Note that there may be auto-oscillations stipulated by the feedback in a hydraulic part of the system due to the fact that the system is located on a stand.

Experiments showed that, in certain cases, there may be a significant increase of the amplitude of auto-oscillations of the stand. Thus, the oscillation frequencies of the liquid in the tank are characterized by the eigenfrequencies of the stand that coincide with the eigen-oscillations of the output pressure. The amplitude of the oscillations may have white-noise-like-behaviour.

The main problem in the study of such systems is to describe the deviation of the system state from the equilibrium.

3.1. Dynamics of fluid in the pipeline. In the one-dimensional case, the dynamics of a compressed liquid in the homogeneous pipeline is usually described by a system consisting of the following equations:

A) Equation of motion of the liquid:

$$
\frac{\partial V}{\partial t}+u \frac{\partial V}{\partial x}+\frac{1}{\gamma} \frac{\partial p}{\partial x}+\frac{\lambda}{2 d} V|V|=0 .
$$

Here, $\lambda$ is the resistance coefficient depending on the Reynolds number, $x$ represents the coordinate of an axis of the pipeline, $\gamma$ is the density of the liquid, $d$ is the diameter of the pipeline, $p, V$ are the instantaneous pressure and speed of the liquid, respectively.

B) Equation of continuity:

$$
\frac{\partial \gamma}{\partial t}+V \frac{\partial \gamma}{\partial x}+\gamma \frac{\partial V}{\partial x}=0
$$


C) The equation of state for the liquid, whose role is played by Hook's law, is

$$
\gamma=\gamma_{0}\left(1+\frac{p-p_{0}}{\gamma_{0} c^{2}}\right)
$$

For the majority of hydraulic systems, the convection terms can be neglected. For the turbulence analysis, the nonlinear term admits linearization, and the equations with the distributed parameters reduce to the form

$$
\begin{gathered}
-\frac{\partial p}{\partial x}=\frac{\gamma}{F_{\tau}}\left(\frac{\partial q}{\partial t}+k q\right), \\
\frac{\partial p}{\partial t}=\frac{c^{2} \gamma}{F_{\tau}} \frac{\partial q}{\partial x} .
\end{gathered}
$$

Here, $q$ is the volumetric intensity of the liquid.

3.2. The description of the centrifugal pump and pressure head sprocket. Among the main characteristic features describing the mode of operation of the cavitational centrifugal pump is the dependence of the sizes of the cavitational cavities $V_{k}$ on the input and output pressure $V_{k}=f(p, q)$. This dependence is related to the cavitation number $k\left(V_{k}, q\right)$ defined as the ratio of the pressure difference to the velocity head $p=p_{n}+k\left(V_{k}, q\right) \gamma \frac{\omega^{2}}{2}$, where $p_{n}$ is the pressure of the saturated steam of the liquid, $\omega$ is the speed of the blade-to-blade flow in the auger channels, $\gamma$ is the density of the liquid steam. When the liquid passes through the auger, the pressure varies according to the rule

$$
p_{n}=\left(a_{i} n^{2}+b_{i} n q^{(1)}+c_{i}\left(q^{(1)}\right)^{2}\right) f_{1}(V),
$$

where $n$ is the number of revolutions of the shaft, $a_{i}, b_{i}, c_{i}$ are the parameters describing the operation of the pump and the pressure head sprocket, $f_{1}(V)$ is an experimentally obtained function describing the influence of the size of the cavitational cavities on the operation of the auger of the pump and the pressure of the head sprocket.

Thus, a simplified model of the dynamics of the system is as follows:

$$
\begin{gathered}
\text { tank } \rightarrow \text { delivery pipeline } \rightarrow \text { auger of the pump } \rightarrow \text { pressure head pipeline } \rightarrow \\
\text { chamber of combustion positioned on the sliding stand. }
\end{gathered}
$$

This reduces to the following mathematical problems.

3.3. Model without sharing cavitational cavities. The corresponding system contains the following equations:

1) Equation of mechanical oscillations of the stand:

$$
\ddot{z}_{i}+b_{i}(t) \dot{z}_{i}+\omega_{i}^{2}(t) z_{i}=\frac{R(t)}{m(t) \bar{p}_{6}} p_{6}, \quad i=1,2, \ldots, 6 ;
$$

2) Equation of motion of the liquid in the pipeline:

$$
-\frac{\partial p_{1}}{\partial x}=\frac{\gamma_{1}}{F_{1}} \frac{\partial q_{1}}{\partial t}
$$




$$
-\frac{\partial p_{1}}{\partial t}=\frac{c^{2} \gamma_{1}}{F_{1}} \frac{\partial q_{1}}{\partial x}
$$

with the boundary conditions

$$
\begin{gathered}
p_{1}(0, t)+R_{1} q_{1}(0, t)=\frac{\gamma_{1} H(t)}{g} \sum_{i=1}^{6} \alpha_{i l} \ddot{z}_{i}+\beta \gamma_{1} F_{1} \sum_{i=1}^{6} \alpha_{i 2} \dot{z}_{i}, \\
p_{1}\left(l_{1}, t\right)=B_{1} V_{k}+B_{2} q_{1}\left(l_{1}, t\right)+E \dot{V}_{k}+I_{k} \dot{q}\left(l_{1}, t\right) .
\end{gathered}
$$

Here, $l_{1}$ is the length of the pipeline and, in the quasistationary model, $E=I_{k}=0$.

3) Equation of motion of the liquid in the pressure head pipeline:

with the boundary conditions:

$$
\begin{gathered}
-\frac{\partial p_{2}}{\partial x}=\frac{\gamma_{2}}{F_{2}} \frac{\partial q_{2}}{\partial t} \\
-\frac{\partial p_{2}}{\partial t}=\frac{c^{2} \gamma_{2}}{F_{2}} \frac{\partial q_{2}}{\partial x}
\end{gathered}
$$

$$
\begin{gathered}
p_{2}\left(l_{1}+0, t\right)+R_{2} q_{2}\left(l_{1}+0, t\right)=p_{1}\left(l_{1}-0, t\right)+s_{1} q_{1}\left(l_{1}-0, t\right)+\varepsilon V_{k}, \\
\tau \dot{p}_{2}\left(l_{1}+l_{2}, t\right)+p_{2}\left(l_{1}+l_{2}, t\right)=A_{2} q_{2}\left(l_{1}+l_{2}, t\right), \\
p_{2}\left(l_{1}+l_{2}, t\right)=p_{2}(t) .
\end{gathered}
$$

4) Equation of material balance describing summarized size of cavitational cavities in the pump:

$$
\gamma_{1} \dot{V}_{k}=q_{2}\left(l_{1}+0, t\right)-q_{1}\left(l_{1}-0, t\right) .
$$

Relations (3.1), (3.2) can be considered to be the impulse effect concentrated on the curves transversal to the characteristics. Thus, we have to study solutions of the hyperbolic equations with impulse effect concentrated on surfaces transversal to characteristics $[18,19]$.

3.4. Reduction to the integral equations and inequalities. The reduced model is represented by an equation of hyperbolic type with, generally speaking, a nonlinear right-hand member and boudary conditions acting as the impulse effect.

Let us assume $s_{i}=c_{i}$ and $z_{i}=\frac{c_{i} F_{i}}{\gamma_{i}}$. The general solution of the partial differential equations considered has the form $p_{i}(x, t)=\nu\left(x-s_{i} t\right)+u\left(x+s_{i} t\right)$ and $q_{i}(x, t)=$ $\frac{1}{z_{i}}\left(\nu\left(x-s_{i} t\right)-u\left(x+s_{i} t\right)\right)$. In view of the boundary conditions at $t=0$, by making the standard substitution $\xi=x-s t$, and $\eta=x+s t$, applying the d'Alembert formula in the domain $0<\xi+\eta<2 l_{1}$, we obtain the following representation of the solutions:

$$
u_{1}(\xi, \eta)=\frac{\varphi_{1}(\xi)+\varphi_{1}(\eta)}{2}+\frac{1}{2 z_{i}} \int_{\xi}^{\eta} \psi_{1}(s) d s+\frac{z_{i}}{2} \iint_{D} f_{1}(s, t) u_{1}(s, t)\left|u_{1}(s, t)\right| d s d t .
$$

Here, the functions $\varphi, \psi$ are determined from the initial and boundary conditions. The integration is carried out in the domain

$$
D_{1}=\left\{s, t: s+t>0, \quad s<\xi, \quad t<\eta, \xi+\eta<2 l_{1}\right\} .
$$


Similarly, in the domain $2 l_{1}<\xi+\eta<2 l_{2}$, the formula of representation for solutions has the form

$$
\begin{aligned}
u_{2}(\xi, \eta)=\frac{\varphi_{2}(\xi)+\varphi_{2}(\eta)}{2}+ & \frac{1}{2 z_{i}} \int_{\xi}^{\eta} \psi_{2}(s) d s+\frac{z_{i}}{2} \iint_{D_{2}} f_{2}(s, t) u_{2}(s, t)\left|u_{2}(s, t)\right| d s d t+ \\
& +\int_{D_{2} \cap \gamma} u(s, t) \varphi_{2}(s, t) d \mu .
\end{aligned}
$$

Here,

$$
D_{2}=\left\{s, t: s+t>0, \quad s<\xi, \quad t<\eta, \quad 2 l_{1}<\xi+\eta<2 l_{2},\right\}
$$

the curve $\gamma=\left\{\xi, \eta: \xi+\eta=2 l_{1}\right\}$ represents the curve of rupture of the stream stipulated by the presence of the pump. The functions are constant along the characteristics in the domain $D_{2} \backslash D_{1}$; their change is determined by a Stieltjes integral on the curve $\gamma$ and depends on the constant characteristics of the pump. We do not consider the process of reflection of a wave from the blades of the pump.

Thus, the problem considered can be studied by using the multi-dimensional integral inequalities considered in Section 2.

\section{REFERENCES}

[1] Samoilenko, A. M. and Perestyuk, N. A.: Differential Equations with Impulse Effect, Vyshcha Shkola, Kyiv, 1987. (in Russian)

[2] Samoilenko, A., Borysenko, S., Cattani C. and Yasinsky V.: Differential Models: Construction, Representations 83 Applications, Nauk. Dumka, Kyiv, 2001.

[3] Samoilenko A., Borysenko, S., Cattani, C., Matarazzo, G. and Yasinsky, V.: Differential Models: Stability, Inequalities \& Estimates, Nauk. Dumka, Kyiv, 2001.

[4] Samoilenko, A. M. and Borysenko, S. D.: Integro-sum inequalities and stability of processes with discrete disturbances, Proc. of the Third International Conference "Differential Equations and Their Applications", Russia, (1987), 377-380.

[5] Samoilenko, A. M. and Borysenko, S. D.: On functional inequalities of Bihari type for Discontinuous functions, Uspekhi Mat. Nauk, 53(4), (1998), 147-148.

[6] Borysenko, S. D., Kosolapov, V.I. and Obolensky A.Yu.: Stability of Processes under Continuous and Discrete Perturbations, Nauk. Dumka, Kyiv, 1988. (in Russian)

[7] Borysenko, S. D.: Construction of Mathematical Models, NTUU "KPI"(National Technical University "Kiev, Polytechnical Institute"); (1995). (in Russian)

[8] Borysenko, S. D.: Integro-sum inequalities for functions of many independent variables, Differents. Uravneniya, 23(9), (1989), 1638-1641.

[9] Borysenko, S. D.: On some integro-sum inequalities and their applications, Vest. Kiev. Univ. Mat. i Mekh., 31, (1989), 7-12.

[10] Borysenko, S. D.: On integro-sum functional inequalities, Differents. Uravneniya, 34(6), (1998), p. 850.

[11] Borysenko, S. D.: On some Bihari inequalities for discontinuous functions, Nauk. Visti NTUU "KPI", 1 (1998), 147-151.

[12] Borysenko, S. D.: Multidimensional integro-sum inequalities, Ukr. Nat. Zh., 50, No. 2 (1998), 172-177. 
[13] Borysenko, S. D.: Integro-sum inequalities and stability problems of systems with impulse excitation, Author's Abstract of the DSc Dissertation (Phys. \& Math.), Inst. of Math., NAS of Ukraine, Kyiv, 1998.

[14] Borysenko, S. D., Matarazzo, G. and Piltyay M. M.: One-dimensional integro-sum inequalities. The Bellman-Bihari-Rakhmatullina method, Nauk. Visti NTUU "KPI", 1 (2000), 111-115.

[15] Borysenko, S. D., Matarazzo, G. and Piltyay M. M.: Problem of reduction of multidimensional functional integral inequalities to one-dimensional ones, Neliniyni Kolyvannya, 2, (2000), 18-24.

[16] Slyusarchuk, V. E.: General theorems on existence solutions of differential equations with impulse influence, Ukr. Mat. Zh., 52(7), (2000), 954-964.

[17] Pilipenko, V. V., Zadontsev, V. A. and Natanzon M. S.: Cavitational AutoOscillations and Dynamics of Hydrosystems, Mashinostroenie, Moscow, 1977. (in Russian)

[18] Pilipenko, V. V., Zadontsev, V. A. and Chalyi N. P.: Theoretical Stability Investigation of the Auger - Centrifugal Pump - Pipeline System for Systems on the Stand. Cavitational Auto-Oscillations and Dynamics of Hydrosystems, Nauk. Dumka, Kiev, 1977. (in Russian)

[19] Hale, J.: Theory of Functional-Differential Equations, Mir, Moscow, 1984.

[20] Kolmanovsky, V. G. and Nosov, V. P.: Stability and Periodic Solutions of Delay Systems, Nauka, Moscow, 1981. (in Russian)

[21] Martinyuk, A. A., Obolensky, A. Yu. and Chernitskaya L. N.: Mathematical models of hydromechanical systems, in: Stability of Hydromechanical Systems, Inst. of Mechanics, Akad. Nauk Ukr. SSR, Dep. VINITI (1986), 5-36. 